\title{
Caracterização nutricional de idosos com hipertensão arterial em Teresina, PI.
}

\section{Nutritional characterization of elderly people with hypertension in Teresina, Piauí State.}

\section{Resumo}

O objetivo do estudo foi avaliar o estado nutricional e o consumo alimentar, e caracterizar aspectos do estilo de vida de idosos hipertensos que freqüentam centros de convivência na cidade de Teresina-PI. O estudo, do tipo transversal, envolveu 125 idosos, de ambos os sexos (17 homens e 108 mulheres), avaliados por meio de questionário previamente testado para a caracterização da casuística. As variáveis nutricionais foram investigadas por antropometria, medidas de circunferência e pelo levantamento do consumo alimentar nas últimas 24 horas. Encontraram-se índices elevados de sobrepeso $(45,6 \%)$ e baixo-peso $(36,0 \%)$, a pressão arterial diastólica $(\mathrm{PAD})$ associando-se positivamente com o sobrepeso (índice de massa corporal $27 \mathrm{~kg} / \mathrm{m}^{2}$ ). A distribuição da gordura central apresentou elevada prevalência (88\%), indicando risco para doenças cardiovasculares. Os alimentos mais consumidos foram: feijão, leite, pães, cereais, arroz, massas e carnes. Não se observou grande adesão às medidas terapêuticas não-farmacológicas no grupo de idosos hipertensos, o que mostra a necessidade de rever as orientações propostas no Programa de Controle da Pressão Arterial para o Idoso.

\section{Abstract}

The objective of this study was to evaluate the nutritional state and food consumption, and to characterize some aspects of life style, of hypertensive elderly who attend two social centers in Teresina city. The cross-sectional study

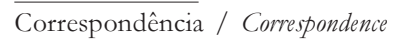

Cecília Maria Resende Gonçalves de Carvalho

Universidade Federal do Piauí - Campus Universitário Ministro Petrônio Portela, Centro de Ciências Humanas e Letras, Núcleo de Pesquisa e Extensão Universitária para a Terceira Idade, Sala 335, Bairro Ininga 64049-550 Teresina, PI - Brasil

E-mail: nupeuti@ufpi.br
Palavras-chave: idoso; hipertensão; estado nutricional; nutrição do idoso; consumo de alimentos; Teresina, Pi 
comprised 125 elderly individuals of both sexes (17 men and 108 women), who were interviewed through a previously tested questionnaire. Nutritional variables were assessed through anthropometry, circumference measures and a 24 -hour food record. The analysis found high rates of overweight $(45,6 \%)$ and low weight $(36,0 \%)$. The diastolic arterial pressure was positively associated with overweight (body mass index $>27$ ). The distribution of central lipids showed high prevalence, indicating risk for cardiovascular diseases. The most consumed food types were: beans, milk, breads, cereals, rice, pastas and meats. A great adhesion to non-pharmacologycal therapies in the group of hypertensive elderly was not observed, showing the need to review the guidelines of the Control of Arterial Pressure Program for the Elderly.
Word Key: aged;

hypertension; nutritional status; elderly nutrition; food consumption;

Teresina,pi city

\section{INTRODUÇÃO}

O envelhecimento populacional é, hoje, uma realidade mundial. No Brasil, o número absoluto de pessoas com mais de 60 anos aumentou nove vezes nas últimas seis décadas. Em 1940 era de 1,7 milhões e em 2000 saltou para 14,5 milhões, projetando-se para 2020 um contingente de aproximadamente 30,9 milhões de pessoas com mais de 60 anos (Beltrão, Camarano \& Kanso ${ }^{1}$, 2004).

O crescimento da população idosa é conseqüência da queda da fecundidade e da mortalidade, que torna esse grupo populacional um componente importante e cada vez mais expressivo dentre a população geral. Conseqüentemente, as mudanças na estruturação etária da população implicam um aumento na incidência e prevalência de doenças crônicas não-transmissíveis, com $85 \%$ das pessoas com 60 anos ou mais apresentando pelo menos uma doença crônica (Ramos ${ }^{20}$ et al, 1987).

Entre as doenças crônicas, as cardiovasculares constituem a principal causa de morte na pessoa idosa, pois podem gerar incapacidades, dependências e perda de autonomia, representando um alto custo econômico e social. Nesse contexto, a hipertensão arterial sistêmica é a mais prevalente e aumenta progressivamente com a idade (Santos $^{21}$ et al., 2002).

O Estudo Multicêntrico do Idoso (EMI) demonstrou uma prevalência de $65 \%$ na população idosa; entre as mulheres com mais de 75 anos, a prevalência de hipertensão pode chegar a $80 \%$ (Brandão ${ }^{1}$ et al, 2002). Dados do Framingham Heart Study demonstraram que indivíduos que atingem os 65 anos sem HAS têm $90 \%$ de chance de se tornarem hipertensos (Miranda $\left.{ }^{16}, 2005\right)$.

A HAS está associada a complicações bastante freqüentes em idosos, como doença arterial coronariana, doença cérebro-vascular, insuficiência cardíaca, insuficiência renal crônica, entre outras. $\mathrm{O}$ alto custo social dessa doença é responsável por cerca de $40 \%$ dos casos de aposentadoria precoce e de absenteísmo no trabalho (Consenso ${ }^{8}$ 1999). 
As modificações que vão ocorrendo com o envelhecimento acarretam importantes alterações cardiovasculares, o que explica a freqüente associação da hipertensão arterial na pessoa idosa. A probabilidade de incidência da HA é influenciada por condições multicausais que, além de aspectos como sexo, raça, idade, hereditariedade - que certamente não podem sofrer intervenção (Cabral $\left.{ }^{5}, 2003\right)$ - podem ser determinadas também por alguns fatores plenamente modificáveis, como sobrepeso, ingestão excessiva de sal, sedentarismo, consumo de álcool, tabagismo, condições socioeconômicas, dislipidemias, dentre outras $\left(\mathrm{SESAPE}^{22}, 2002\right)$.

O tratamento da HA envolve orientações para que ocorram mudanças de hábitos de vida, compreendendo o tratamento nãomedicamentoso e o tratamento com agentes anti-hipertensivos.

O Governo Federal, através do Ministério da Saúde, vem desenvolvendo ações de forma a intervir no comportamento de dois agravos à saúde - hipertensão arterial e diabetes mellitus - com o propósito de vincular os portadores dessas patologias às unidades de saúde, garantindo-lhes acompanhamento e tratamento sistemático (Brasil³ , 2002a).

Dessa forma, analisar as condições de saúde nutricional do idoso hipertenso, identificando o número de fatores de risco a que esses indivíduos estão expostos, é de grande interesse em saúde pública.

Para alcançar as metas propostas pela Agenda Nacional de Prioridades de Pesquisa em Saúde do Ministério da Saúde, com o tema "Saúde do Idoso", este estudo se propõe a investigar, num grupo de idosos hipertensos que freqüentam dois centros de convivência na cidade de Teresina, as condições nutricionais que envolvem os aspectos antropométricos e de consumo alimentar, associados a outros fatores de estilo de vida. Vale ressaltar a importância da pesquisa, na medida em que prioriza caracterizar o estilo de vida desses indivíduos, que é condição importante para a redução da mortalidade por DAC, visando a modificar os fatores de risco que favoreçam o controle e a redução da hipertensão arterial.

\section{METODOLOGIA}

O estudo, de desenho observacional, transversal e descritivo, foi realizado em dois centros de convivência de Teresina, Piauí, local de entretenimento, lazer, realização de palestras e de prestação de serviços que se destina ao atendimento em saúde de pessoas com idade a partir de 60 anos, apoiados pelo Programa de Hipertensão Arterial da Prefeitura Municipal de Teresina.

Foram incluídos no estudo os indivíduos com idade igual ou superior a 60 anos, com hipertensão arterial, sem comprometimento de memória que prejudicasse as informações a serem investigadas, com participação voluntária e com assinatura ou impressão digital em termo de consentimento livre e esclarecido, após orientação quanto aos objetivos e procedimentos da pesquisa. 
Dos 150 idosos hipertensos matriculados e acompanhados nos dois centros no ano de 2005, 125 representaram o número amostral mínimo. Eles concordaram em participar espontaneamente do estudo, com uma estimativa de $10 \%$ de expectativa de freqüência na população teresinense e probabilidade de 0,05 para efeito do erro alfa.

Antes de dar prosseguimento ao levantamento dos dados e avaliação do estado nutricional, a aferição da pressão arterial foi realizada em todos os participantes, seguindo-se o protocolo proposto pelo III Consenso Brasileiro de Hipertensão Arterial: ambiente calmo, com a pessoa idosa relaxada, sentada (pernas não cruzadas), com o braço desnudo apoiado em uma superfície plana. Com o estetoscópio e o tensiômetro regulados, colocava-se o manguito cerca de 2 ou 3 $\mathrm{cm}$ acima da fossa anticubital; posicionando o estetoscópio sobre a artéria braquial, o manguito foi inflado até o desaparecimento da pulsação, prosseguindo com a deflação do ar com velocidade constante até o aparecimento do primeiro batimento (pressão sistólica), que persistiu até o nível zero (pressão diastólica). Após um a dois minutos, o procedimento foi repetido, e a média dos valores anotados $\left(\mathrm{SBH}^{24}, 2002\right)$.

Para a descrição da casuística e determinação dos fatores de estilo de vida, foram obtidas informações mediante a aplicação de questionário desenvolvido com essa finalidade.

Para a determinação do índice de massa corporal (IMC), foram tomadas medidas de peso corporal e estatura. O peso corporal foi obtido em balança antropométrica tipo plataforma, com precisão de $100 \mathrm{~g}$, da marca Filizola, estando os idosos descalços e com o mínimo de roupa possível. Para aferição da estatura utilizou-se o antropômetro fixo da mesma balança com precisão em milímetros, sendo mantido o indivíduo em posição ortostática, com os pés juntos.

$\mathrm{Na}$ classificação do estado nutricional, utilizou-se o índice de massa corporal, $\left[\mathrm{IMC}=\operatorname{peso}(\mathrm{kg}) / \operatorname{altura}(\mathrm{m})^{2}\right]$, considerando-se a seguinte classificação: $<22$ desnutrição, de 22 a 27 peso adequado e $>27$ sobrepeso (Lipschitz $^{15}$, 1994).

A circunferência da cintura foi mensurada com o idoso em posição ereta, abdômen relaxado, braços entendidos ao longo do corpo e os pés separados a uma distância de 25$30 \mathrm{~cm}$. A roupa do idoso foi afastada, de forma que a região da cintura ficasse despida, e a medida foi então aferida com fita métrica inextensível com escala de 0,5 centímetros, colocada sem fazer pressão, em plano horizontal ao nível da cintura natural. $\mathrm{Na}$ impossibilidade desta identificação, considerou-se a menor circunferência entre a porção inferior da última costela e a crista ilíaca (Cecan/ $\mathrm{Rco}^{7}$, 2003; Brasil $\left.^{4}, 2004 b\right)$.

A medida da circunferência da cintura como mais um dado analisado permitiu-nos detectar a obesidade central e identificar o risco para doenças ateroscleróticas, utilizando como parâmetros de risco aumentado para população feminina e masculina os seguintes valores (SBC $\left.{ }^{23}, 2001\right)$ : para mulheres, risco aumentado e $80 \mathrm{~cm}$ e muito aumentado e $88 \mathrm{~cm}$; 
para homens, risco aumentado e $94 \mathrm{~cm}$ e muito aumentado e $102 \mathrm{~cm}\left(\mathrm{WHO}^{28}, 1997\right)$.

As medidas antropométricas e de circunferência foram realizadas três vezes; em caso de diferença entre duas medidas maior que um centímetro ou 0,5 quilograma, todas foram repetidas.

Foi utilizado como instrumento de medida do consumo alimentar o Questionário de Freqüência de Consumo Alimentar-QFCA, para verificar a freqüência diária, semanal e mensal, juntamente com o Recordatório de 24 horas. Os instrumentos foram testados na etapa piloto e aplicados numa amostra composta por dez idosos, dois recordatórios de 24 horas, por participante, em dias distintos, incluindo um dia do final de semana, para levantar o consumo alimentar nas últimas 24 horas que antecederam a entrevista, os quais fizeram parte da lista de alimentos do QFCA.

Além do QFCA, foi utilizada também a Pirâmide Alimentar (Phillipi ${ }^{18}$, 1999) como instrumento de orientação nutricional, objetivando a realização de uma análise comparativa entre o consumo alimentar qualitativo e quantitativo recomendado por esse instrumento e os resultados encontrados neste estudo.

Os dados foram processados no programa EPI-INFO $6.04^{10}$, para a obtenção de ta- belas simples e cruzadas. Realizou-se, ainda, a Análise de Variância (ANOVA) e aplicaramse os testes qui-quadrado para as variáveis categóricas, o teste $T$ para variáveis contínuas e Kruskall-Wallis para análise das variações dos dados coletados.

O estudo foi aprovado pelo Comitê de Ética em Pesquisa da UFPI (Protocolo: 0017.0.045.000-05).

\section{RESULTADOS}

Foram avaliados 125 indivíduos de $60 \mathrm{a}$ 91 anos, com idade média de 71,7 anos. A distribuição entre as diversas faixas etárias (tabela 1) revelou que 52 indivíduos $(41,6 \%)$ estavam entre 60 a 69 anos; 58 (46,4\%) entre 70 a 79 anos e $15(12 \%)$ com 80 anos ou mais. Em relação à distribuição por gênero, esta pesquisa constatou um predomínio bastante expressivo do sexo feminino $(86,4 \%) \mathrm{em}$ relação ao sexo masculino $(13,6 \%)$. Santos ${ }^{21}$ (2002), em seu estudo, confirma a tendência relacionada com idosos em centro de convivência, em que a participação masculina raramente ultrapassa $20 \%$, de forma que no Brasil a velhice é pensada como uma experiência essencialmente feminina. 
Tabela 1 - Distribuição das características de identificação e de estilo de vida apresentadas pelos idosos pesquisados, Teresina PI. Agosto 2005

\begin{tabular}{|c|c|c|}
\hline \multirow[t]{2}{*}{ Características } & \multicolumn{2}{|c|}{ Freqüência } \\
\hline & $\mathrm{N}^{\mathrm{o}}$ & $\%$ \\
\hline Total de Participantes & 125 & 100,0 \\
\hline \multicolumn{3}{|l|}{ Sexo } \\
\hline Masculino & 17 & 13,6 \\
\hline Feminino & 108 & 86,4 \\
\hline \multicolumn{3}{|l|}{ Faixa Etária (anos) } \\
\hline $60-69$ & 52 & 41,6 \\
\hline $70-79$ & 58 & 46,4 \\
\hline 80 anos ou mais & 15 & 12,0 \\
\hline \multicolumn{3}{|l|}{ Escolaridade } \\
\hline Analfabeto & 38 & 30,4 \\
\hline 1 a 4 anos de estudo & 57 & 45,6 \\
\hline 4 a 8 anos de estudo & 23 & 18,4 \\
\hline 9 anos ou mais & 7 & 5,6 \\
\hline \multicolumn{3}{|l|}{ Estilo de vida } \\
\hline \multicolumn{3}{|l|}{ Fumo } \\
\hline Não & 120 & 96,0 \\
\hline Sim & 5 & 4,0 \\
\hline \multicolumn{3}{|l|}{ Etilismo } \\
\hline Não & 124 & 99,2 \\
\hline Sim & 1 & 0,8 \\
\hline \multicolumn{3}{|l|}{ Uso de medicamento } \\
\hline Não & 121 & 96,8 \\
\hline $\operatorname{Sim}$ & 4 & 30,0 \\
\hline \multicolumn{3}{|l|}{ Faz dieta orientada } \\
\hline Não & 35 & 35,0 \\
\hline Sim & 65 & 65,0 \\
\hline \multicolumn{3}{|l|}{ Prática de atividade física } \\
\hline Não & 29 & 23,4 \\
\hline Sim & 95 & 76,6 \\
\hline \multicolumn{3}{|l|}{ Tipo } \\
\hline Caminhada & 18 & 18,8 \\
\hline Hidroginástica & 3 & 3,1 \\
\hline
\end{tabular}


Alguns fatores têm sido atribuídos para justificar a superioridade, em números absolutos, de mulheres idosas no Brasil, quando confrontadas com homens de 65 anos ou mais. Esses fatores foram observados por Berquó (1996 apud Santos ${ }^{21}$, 2002), para quem essa situação decorre da existência da mortalidade diferenciada por sexo, que prevalece há muito tempo na população brasileira, em que a mulher vive oito anos a mais que o homem. A menor exposição a determinados fatores de risco, notadamente no trabalho; a menor prevalência de tabagismo e ao uso de álcool; as diferenças quanto à atitude em relação a doenças e incapacidades e a maior assistência gineco-obstetra foram relatados por $\operatorname{Veras}^{27}$ (1987) como sendo também responsáveis pela maior longevidade feminina em relação à masculina.

Analisando o grau de escolaridade entre idosos pesquisados (tabela 1), 30,4\% relataram ser analfabetos, percentual considerado elevado e preocupante, pois o analfabetismo expõe essa população a enfrentar dificuldades na tentativa de buscar condições de existência e de sobrevivência, inclusive afetando de forma negativa o tratamento de saúde.

Em 1991 a população de idosos cresceu $1,3 \%$, havendo incremento, em 2002, para $1,7 \%$. O índice de analfabetismo vem decrescendo, considerando que, em 1991, 44\% dos idosos não sabiam ler e em 2002 este índice se reduziu para $33,3 \%$. Na tentativa de diminuir os índices de analfabetismo e oferecer melhores condições de vida à pessoa idosa, os centros de convivência vêm mantendo programas de alfabetização para adultos.
A tabela 1 revela, ainda, alguns aspectos do estilo de vida pesquisados, entre eles: o uso do fumo, o consumo de bebida alcoólica, a prática de atividade física, o consumo alimentar e o uso de medicamento.

Quando investigados sobre o uso do tabaco e do álcool, 96\% e 99,2\% negaram a prática dos respectivos hábitos; no entanto, alguns referiram utilização dos mesmos no passado. $\mathrm{O}$ abandono desses hábitos pode estar associado à adesão ao tratamento antihipertensivo. Segundo as IV Diretrizes Brasileiras de Hipertensão Arterial (SBH $\left.{ }^{24}, 2002\right)$, em avaliação por medida ambulatorial da pressão arterial (MAPA), observou-se que a pressão sistólica de hipertenso fumante foi significativamente mais elevada do que em nãofumantes, revelando o importante efeito transitório do fumo. Mostrou ainda o consumo elevado de bebida alcoólica, o qual está associado também à elevação da pressão arterial.

Nos centros de convivência estudados, não foram constatados programas específicos de combate ao tabagismo ou alcoolismo; contudo, na abordagem ao paciente hipertenso, durante a consulta médica observou-se o aconselhamento no sentido de limitar ou abolir o uso de bebidas alcoólicas e o abandono do hábito de fumar.

No tocante à prática sistemática da atividade física, 76,0\% dos idosos revelaram adesão aos programas desenvolvidos no centro. As modalidades de maior preferência foram dança (62,5\%), seguida da caminhada (18\%). Mais da metade (55,8\%) tinha uma freqüência semanal de duas a três vezes, seguidos de 
25,3\% com práticas diárias e 18,9\% apenas de uma vez. Essas atividades foram oferecidas apenas pelo Centro de Convivência da Terceira Idade (CCTI).

A classificação do estado nutricional, segundo o índice de massa corporal (IMC), revelou que 45, $6 \%$ dos idosos tinham sobrepeso, $36,0 \%$ apresentaram baixo-peso e $18,4 \%$ eram eutróficos.

A associação entre o sobrepeso e risco cardíaco (figura 1), representada pela avaliação da circunferência da cintura, mostrou um percentual elevado de idosos com risco au- mentado $(18,4 \%)$ e muito aumentado $(69,6 \%)$. Enquanto o risco de morbidade se torna maior ao aumentar a severidade da obesidade, sabe-se também que o padrão de distribuição da gordura corporal é um indicador ainda mais importante do risco que a obesidade representa à saúde. A medida de circunferência da cintura mostra-se como uma medida regional capaz de fornecer estimativa da gordura abdominal, diagnosticando a obesidade andróide, que, por sua vez, está correlacionada com a gordura visceral, mais associada a distúrbios metabólicos e riscos cardiovasculares (Cuppari', 2005).

Figura 1 - Distribuição dos idosos pesquisados segundo risco de complicações metabólicas associadas ao sobrepeso - Teresina-PI. Agosto de 2005.

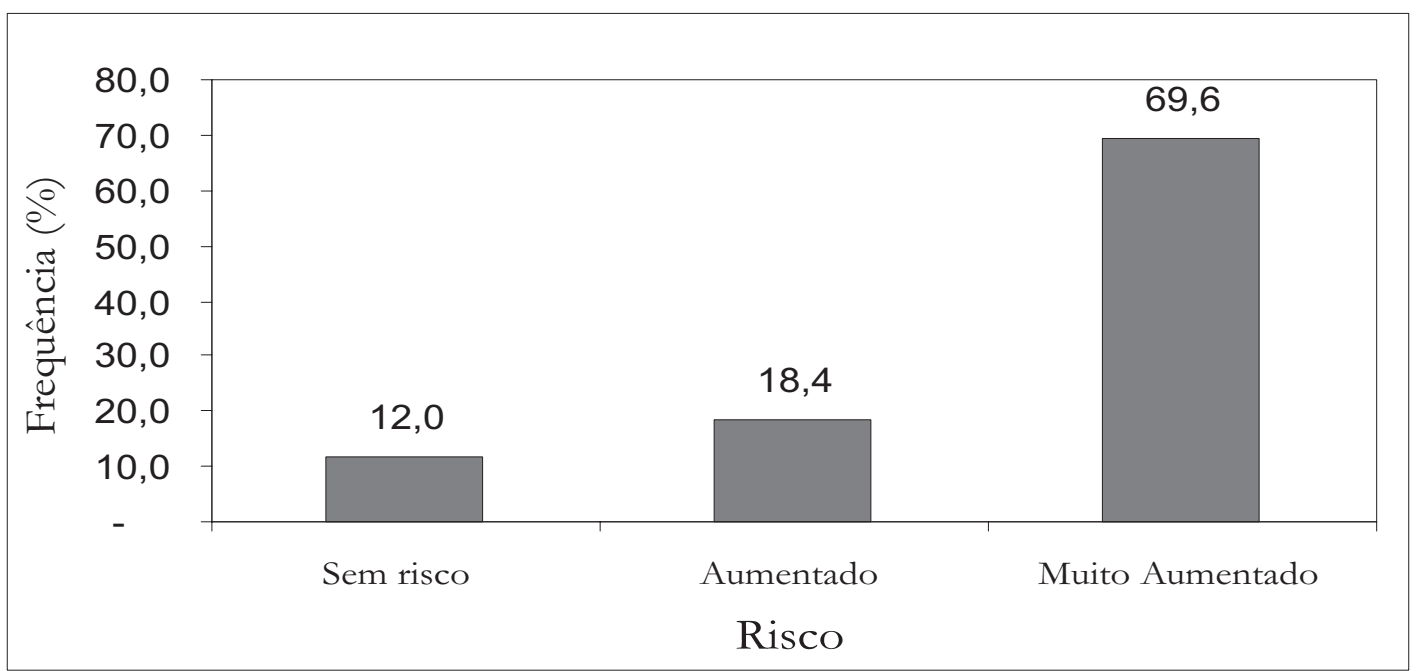


$\mathrm{Na}$ associação entre a prática de atividade física e pressão arterial diastólica - PAD (tabela 2), observou-se que 67,2\% tinham PAD alterada, mesmo nos idosos com prática regular. Provavelmente os programas de exercício promovidos pelos centros não se- jam voltados para a prevenção e controle da hipertensão. As atividades físicas oferecidas pelos centros são classificadas como leve e têm como objetivos: relaxamento, socialização e lazer.

Tabela 2 - Distribuição dos idosos pesquisados, segundo estado nutricional e pressão diastólica, Teresina PI. Agosto 2005

\begin{tabular}{|c|c|c|c|c|c|c|c|c|}
\hline \multirow{2}{*}{$\begin{array}{l}\text { Estado Nutricional } \\
\text { Pressão Diastólica }\end{array}$} & \multicolumn{2}{|c|}{ Baixo Peso } & \multicolumn{2}{|c|}{ Eutrófico } & \multicolumn{2}{|c|}{ Sobrepeso } & \multicolumn{2}{|c|}{ Total } \\
\hline & $\mathrm{n}$ & $\%$ & $\mathrm{n}$ & $\%$ & $\mathrm{n}$ & $\%$ & $\mathrm{~N}$ & $\%$ \\
\hline Normal & 7 & 5,6 & 10 & 8 & 5 & 4 & 22 & 17,6 \\
\hline Alterada & 16 & 12,8 & 35 & 28 & 52 & 41,6 & 103 & 82,4 \\
\hline Total & 24 & 18,4 & 45 & 36 & 57 & 45,6 & 125 & 100 \\
\hline
\end{tabular}

Segundo as IV Diretrizes Brasileiras de Hipertensão Arterial, (SBH $\left.{ }^{24}, 2002\right)$, os programas para prevenção primária ou secundária de doenças cardiovasculares devem contar com atividades aeróbias dinâmicas, tais como: caminhadas rápidas, corridas leves, natação e ciclismos, com freqüência de 3 a 6 vezes por semana, intensidade moderada e sessões de 30 a 60 minutos de duração. Pacientes hipertensos devem iniciar programas de exercício físico regulares, pois, além de diminuir a pressão arterial, ele pode reduzir consideravelmente o risco de doença arterial coronária, acidentes vasculares cerebrais e mortalidade geral.

Com relação ao consumo alimentar, 65\% declararam adesão à dieta (tabela 1); entretanto, a maioria das orientações dietéticas recebidas era fornecida basicamente por médicos, que prestavam alguns aconselhamentos gerais, como reduzir o consumo de sal e gorduras. Não havia preocupação rigorosa em relação à quantidade dos alimentos, uso de temperos naturais com alho, limão, ervas, cebola, ao invés de similares industrializados e outros requisitos necessários a uma alimentação nutricionalmente adequada para o idoso hipertenso. A orientação nutricional é especialmente importante para os idosos, devido às mudanças fisiológicas e aparecimento de doenças relacionadas ao envelhecimento.

Todos os participantes do estudo declararam fazer uso contínuo de medicação para o controle da hipertensão arterial. É importante ressaltar que deve existir uma preocupação em orientar os idosos com relação à interação dos alimentos com medicamentos. Moura ${ }^{17}$ (2002), em sua revisão bibliográfica, ressalta 
que as reações medicamentosas e os problemas nutricionais nos idosos advêm das alterações próprias do processo de senescência e de fatores diversos. Os medicamentos utilizados pelos idosos podem ser mais ou menos absorvidos, dependendo das condições de consumo, ou seja, se associados ou não às refeições, bem como seu estado nutricional. Por outro lado, as deficiências nutricionais podem ocorrer por indução medicamentosa, sendo as mais freqüentes as depleções de vitaminas e minerais.

Neste estudo, a média dos níveis pressóricos encontradas foi 130/90 $\mathrm{mmHg}$, valores que podem ser considerados controlados, segundo a referência para hipertensão das IV Diretrizes Brasileiras de Hipertensão $\left(\mathrm{SBH}^{24}, 2002\right)$. Tais resultados (tabela 3) são atribuídos ao uso contínuo da medicação antihipertensiva e mudanças positivas em alguns aspectos do estilo de vida, como dieta, hábitos de tabagismo e etilismo e atividade física. Com relação à associação da obesidade e hipertensão, amplamente discutida pela literatura mundial, são freqüentes em pessoas obesas ou com sobrepeso níveis pressóricos elevados (Consenso $\left.{ }^{8}, 1999\right)$. No estudo em análise, constatou-se que $41,6 \%$ dos idosos com sobrepeso tinham PAD elevada, evidenciando uma correlação positiva com o estado nutricional.

De acordo com as recomendações do documento Dietary Approaches to Stop Hypertension (the DASH Diet), as quotas diárias por grupo de alimentos devem ser compostas por 7 a 8 porções de grãos ou produtos de grãos, 4 a 5 porções de vegetais, 4 a 5 de frutas, 2 a 3 porções de gorduras e óleos $(27 \%$ das calorias) e não mais que duas porções de carnes, aves ou peixes. Esse estudo multicêntrico controlado (the DASH Diet) mostrou que uma dieta rica em frutas, verduras e grãos, pobre em gorduras saturadas e colesterol pode reduzir a PA (University Of Michigan Health System ${ }^{26}$, 2005).

Quanto aos resultados da freqüência sobre o consumo alimentar dos idosos (tabela 3), observou-se uma freqüência de consumo de 5 a 7 vezes, para os seguintes grupos de alimentos: 66,4\% consumiram leite; 36,0\% carnes bovinas, aves e peixes; 73,6\% feijão; $39,2 \%$ frutas; $52,0 \%$ pães, cereais, arroz e massas; 77,6\% açúcar e 60,8\% café; enquanto com uma menor freqüência, de apenas 2 a 3 vezes por semana, $35,2 \%$ consumiram vegetais e legumes. 
Tabela 3 - Freqüência de grupos de alimentos mais consumidos pelos idosos pesquisados, Teresina PI. Agosto 2005.

\begin{tabular}{llccccc}
\hline Freqüência & \multicolumn{2}{c}{2 a 3 vezes } & \multicolumn{2}{c}{5 a 7 vezes } & \multicolumn{2}{c}{ Total } \\
Grupos de & \multicolumn{2}{c}{ p/semana } & \multicolumn{2}{c}{$\mathrm{p} /$ semana } & $\mathrm{n}$ & $\%$ \\
Alimentos & $\mathrm{n}$ & $\%$ & $\mathrm{n}$ & $\% 3$ & & \\
\hline Leite & 13 & 10,41 & 83 & 66,4 & 96 & 76,8 \\
Carnes Bovinas, Aves, & 28 & 22,4 & 45 & 36,0 & 72 & 57,6 \\
Peixes & & & & & & \\
Feijão & 2 & 1,6 & 92 & 73,6 & 94 & 75,2 \\
Vegetais/Legumes & 44 & 35,2 & 31 & 24,8 & 75 & 60,0 \\
Frutas & 42 & 33,6 & 49 & 39,2 & 91 & 72,8 \\
Pães, Cereais, Arroz e & 29 & 23,2 & 65 & 52,0 & 94 & 75,2 \\
Massas & 1 & 0,8 & 97 & 77,6 & 98 & 78,4 \\
Açúcar & 14 & 11,2 & 86 & 68,8 & 100 & 80,0 \\
Café & 49 & 39,2 & 24 & 19,2 & 73 & 58,4 \\
Chá & 14 & 11,2 & 86 & 68,8 & 100 & 80 \\
Adoçante & & & & & & \\
\hline
\end{tabular}

A hipertensão arterial pode ser controlada por medidas dietéticas específicas que visam não somente a reduzir os níveis pressóricos, mas incorporar hábitos alimentares saudáveis. $\mathrm{Na}$ análise dos grupos de alimentos como fontes de nutrientes associados a ações positivas na redução da pressão arterial, observouse: o leite como fonte de cálcio tem relação entre o seu baixo consumo e ocorrência de hipertensão arterial (Campese \& Bianchi ${ }^{6}$, 1997); o uso de frutas, verduras e leguminosas como fonte de potássio induze a queda da pressão arterial, por aumento da natriurese, e ainda como fonte de fibras. Existem especulações sobre sua capacidade de reduzir a PA não ter sido confirmada pela literatura (Kestin ${ }^{12}$ et al., 1990; Swain ${ }^{25}$ et al., 1990); en- tretanto, seu aumento na dieta pode implicar uma alteração no conteúdo de lipídios, o que certamente levaria a um padrão alimentar mais saudável.

A associação entre o aumento da PA e o consumo de sal é bastante relatada na literatura. Cabral $^{5}$ (2003) descreve em seu estudo a controvérsia que há sobre o efeito do sal na $\mathrm{PA}$, reconhecendo a existência de pessoas que possam ser ou não sensíveis a este elemento na dieta. Considerando as dificuldades de análise individual para essa sensibilidade, recomenda-se a restrição para todos os hipertensos, principalmente pelos outros benefícios que possa trazer, como: menor necessidade de drogas hipotensoras, diminuição da perda de 
potássio induzida por diuréticos, possível redução de hipertrofia do ventrículo esquerdo. Desse modo, a restrição do sódio dietético é um fator a ser bastante considerado. Apesar das limitações na metodologia utilizada para verificar o consumo de sal pelos idosos, os mesmos referiram uma redução de seu uso no preparo dos alimentos, de acordo com as recomendações gerais dos médicos.

Comparando-se a pirâmide alimentar de Philippi (1999), representativa do consumo qualitativo e quantitativo dos alimentos, observou-se que as recomendações por ela estabelecidas não são seguidas no aspecto quantitativo, estando muito aquém do preconizado para o grupo pesquisado; entretanto, elas vêm atendendo de forma qualitativa às necessidades nutricionais.

Outro grupo de alimento que se destacou quanto a sua freqüência foram os açúcares, que podem contribuir para o ganho de peso dos idosos, sendo mais usados em bebidas do que em preparações como sobremesas.

Enfim, o tratamento não-medicamentoso para o controle da HA tem como objetivo diminuir a morbimortalidade cardiovascular, por meio de mudanças no estilo de vida que favoreçam a redução da HA. O controle do excesso de peso corporal, da ingestão de sal, gorduras e de bebidas alcoólicas, assim como a prática regular de exercícios físicos, são as mudanças comprovadamente eficazes na redução e controle de níveis pressóricos, e devem ser realmente incentivadas. No entanto, no grupo de idosos pesquisados não se identificou uma real mudança no estilo de vida, para que fossem alcançadas as metas terapêuticas propostas.

\section{CONSIDERAÇÕES FINAIS}

Os dados encontrados no estudo confirmam a relação entre sobrepeso e a hipertensão arterial em ambos os sexos. Quanto a alguns aspectos de estilo de vida, observaramse mudanças relacionadas ao hábito do tabagismo, etilismo e adesão à prática de atividade física pela maioria dos idosos pesquisados nos dois centros de convivência.

No que se refere aos hábitos alimentares, constataram-se algumas mudanças gerais, como redução na adição de sal e gorduras, e consumo de certas variedades de alimentos, incluindo cereais, leite, verduras e frutas. A ausência, no entanto, do acompanhamento nutricional, inviabiliza o tratamento dietético individualizado, essencial para melhor adesão do paciente e eficácia da terapêutica nãomedicamentosa.

A atividade física desenvolvida nos centros, mesmo não trazendo o benefício da redução de peso corporal, por ser considerada leve e sem fins de gasto energético, pode influenciar positivamente em aspectos como o bem-estar, socialização e promoção de menores níveis de estresse.

As políticas de saúde na área de alimentação dos idosos propõem que os planos de ações nacionais de nutrição reconheçam as pessoas idosas como grupos vulneráveis, e incluam medidas especiais para prevenir a 
desnutrição e garantir políticas alimentares que capacitem indivíduos de todas as idades a escolher alimentos saudáveis.

Considerando a importância da qualidade de vida na terceira idade, tornam-se necessárias políticas públicas multi-setoriais que assegurem um envelhecimento ativo, a fim de melhorar as condições de saúde e aumentar a participação dessa população idosa junto à sociedade.

\section{NOTAS}

a Nutricionista, estudante do Curso de Especialização em Alimentos e Nutrição, Departamento de Nutrição, Universidade Federal do Piauí.

b Nutricionista, especialista em Saúde Pública e em Nutrição Materno Infantil.

c Estatístico, Mestre e Pesquisador na Fundação Municipal de Saúde - Teresina/PI.

d Doutora em Ciência da Nutrição, Professora Adjunta no Departamento de Nutrição, Coordenadora do Núcleo de Pesquisa e Extensão Universitária para a Terceira Idade, Universidade Federal do Piauí.

\section{REFERENCIAS}

1. Beltrão KL, Camarano AA, Kanso S.

Dinâmica populacional brasileira na virada do século XX. Rio de Janeiro: IPEA; 2004.

(Texto pra discussão, 1034)

2. Brasil. Ministério da Saúde. Conselho Nacional de Saúde. Resolução N.196/96 Regulamento pesquisas envolvendo seres humanos. 1996 Out 10

3. Brasil. Ministério da Saúde. Plano de reorganização da atenção à hipertensão arterial e diabetes mellitus: manual de hipertensão arterial e diabetes mellitus. Brasília: 2002a.
4. Brasil. Ministério da Saúde. SISVAN.

Vigilância alimentar e nutricional: orientações básicas para a coleta, processamento, análise de dados e informação em serviços de saúde. Brasília: 2004b.

5. Cabral PC, et al. Avaliação antropométrica e dietética de hipertensos atendidos em ambulatório de um hospital universitário. Revista de nutric'aPo 2003; 16(1): 61-71.

6. Campese VM, Bianchi S. Nutritional factors in hypertension. In: Kopple JD, Massry SG.

Nutritional management of renal disease. Baltimore: Williams \& Wilkins; 1997. p.77-95

7. Centro Colaborador em Alimentação e Nutrição da Região Centro-Oeste/MS/UFG CECAN/RCO. Antropometria, manual de técnicas e procedimentos: vigilância nutricional. 2. ed. rev. atual. Goiânia; 2003.

8. Consenso Brasileiro de Hipertensão Arterial III. Revista brasileira de cardiologia 1999; 1(3).

9. Cuppari L. Guia de Nutrição: nutrição clínica no adulto. 2. ed. Barueri/,SP: Manole; 2005. p. 89-127.

10. Epi-Info. World Health Organization. Center Control Diseases. Version 6.04b, 2001 January.

11. Franken RA, Taddei CFG, coordenadores. Diretrizes do grupo de estudo em cardiogeriatria da Sociedade Brasileira de Cardiologia. Arq Bras Cardiol 2002, 79 (Sup 1):1-45.

12. Kestin M, Moss R, Clifton PM, Nestel PJ. Comparative effects or three cereal brans on plasma lipids, blood pressure and glucose metabolism in mildly hipercholesterolemic men. Am J Clin Nutr 1990; 52 (4): 661-6.

13. Kopple JD, Massry SG. Nutritional management of renal disease. Baltimore: Williams \& Wilkins; 1997. p.77-95. 
14. Krummel D. Nutrição e hipertensão. In: Mahan LK, Escott-Stump S. Krause: alimentos, nutrição e dietoterapia. 10.ed. São Paulo: Roca; 2002a. p. 576 - 90.

15. Lipschitz DA. Screening for nutritional status in the elderly. Primary care 1994; 21(1): 55-67.

16. Miranda RD. Hipertensão arterial: o que muda na fisiopatologia e no tratamento. In: Ramos LR. Geriatria e Gerontologia. Guias de medicina ambulatorial do hospitalar UNIFESP - Escola Paulista de Medicina. 2. ed. São Paulo: Manole; 2005. p.61-75.

17. Moura MRL. Interação fármaco-nutriente: uma revisão. Revista de nutriçaßo 2002; 15 : 223-38.

18. Philippi ST, Latterza AR, Cruz ATR, Ribeiro LC. Pirâmide alimentar adaptada: guia para escolha de alimentos. Revista de nutrição 1999; 12(1): 65-80.

19. Pompeu JGF. O orientador de bolso recomendação para a elaboração de trabalhos acadêmicos. São Paulo: Memnon; 2003.

20. Ramos LR, Veras RP, Kalache A. Envelhecimento populacional: uma realidade brasileira. Revista de Saúde Pública 1987; 21 : 211-24.

21. Santos SR, Santos IBC, Fernandes MGM, Henriques MERM. Qualidade de vida do idoso na comunidade: aplicação da escala de Flanagan. Rev Lat Am Enfermagem 2002; 10: 757-64.

22. Secretaria de Saúde de Pernambuco. Boletim informativo das doenças crônicodegenerativas 2002;2(02)
23. Sociedade Brasileira de Cardiologia. III Diretrizes brasileiras sobre dislipidemias e diretrizes de prevenção da aterosclerose do departamento de aterosclerose da Sociedade Brasileira de Cardiologia 2001; 77(Supl III)

24. Sociedade Brasileira de Hipertensão; Sociedade Brasileira de Cardiologia; Sociedade Brasileira de Nefrologia. IV Diretrizes brasileiras da hipertensão arterial. Campos do Jordão; 2002.31p.

25. Swain J, Rouse IL, Curley CB, Sacks FM. Comparison of the effects of oat bran and low-fiber wheat on serum lipoprotein levels and blood pressure. The New England journal of medicine 1990; 322: 147-54.

26. University of Michigan Health System. Adult Health Advisor. Dietary approaches to stop hypertension (The DASH Diet). Mckesson: 2005. Disponível na: URL: http:// www.med.umich.edu/

27. Veras RP, Ramos LR, Kalache A. Crescimento da população idosa no Brasil: transformações e conseqüências na sociedade. Revista de Saúde Pública 1987; 21(3): 225-33.

28. Word Health Organization. Obesity. Preventing and managing the global epidemic. Geneva: Report of a WHO Consultation on Obesity; 1998.

Recebido para publicação em: 05/05/2006 Aceito em: 15/12/2006 